\title{
Retroperitoneal schwannoma mimicking a pancreatic cystic neoplasm: a case report
}

\author{
Samer AlMasri ${ }^{1}$, Ibrahim Nassour ${ }^{1}$, Aatur D. Singhi ${ }^{2}$, Amer Zureikat ${ }^{1}$, Alessandro Paniccia ${ }^{1}$ \\ ${ }^{1}$ Department of Surgery, University of Pittsburgh Medical Center, Pittsburgh, PA, USA; ${ }^{2}$ Department of Pathology, University of Pittsburgh Medical \\ Center, Pittsburgh, PA, USA \\ Correspondence to: Alessandro Paniccia, MD. Assistant Professor of Surgery, Division of GI Surgical Oncology, University of Pittsburgh Medical \\ Center, 497 Scaife Hall, 3550 Terrace Street, Pittsburgh, PA 15261, USA. Email: panicciaa2@upmc.edu.
}

\begin{abstract}
Schwannomas are mesenchymal tumors that arise from differentiated peripheral nerve Schwann cells and are mostly benign in nature. They tend to occur most frequently in the head and neck regions or in the trunk and flexor surfaces of the upper extremities. However, deeply seated schwannomas can arise in the posterior mediastinum and very rarely in the retroperitoneal space. Owing to the anatomic location, broad differential diagnosis, elusive presentation, and lack of specific radiologic and clinical features, retroperitoneal schwannomas can pose a significant diagnostic challenge. Accurate diagnosis of this rare pathology is usually established based on immunohistochemistry and histopathologic examination following surgical resection. We present a case of an elderly gentleman who underwent surgical resection of a presumed pancreatic cystic neoplasm. However, final histopathologic evaluation demonstrated a retroperitoneal schwannoma with cystic degeneration. The diagnosis was confirmed through a combination of immunohistochemistry and a comprehensive targeted next generating sequencing assay that analyses 161 genes including those that are known to be commonly mutated in a subset of schwannoma tumors. In this report, we highlight the importance of employing the aforementioned novel molecular techniques to improve diagnostic accuracy for this rare clinical entity and ultimately guide surgical planning and aid in patient counseling.
\end{abstract}

Keywords: Retroperitoneal schwannoma; pancreatic cystic neoplasm; molecular analysis; case report

Received: 19 November 2020; Accepted: 26 February 2021; Published: 20 July 2021.

doi: $10.21037 /$ jovs-20-165

View this article at: http://dx.doi.org/10.21037/jovs-20-165

\section{Introduction}

Schwannomas are rare, slow-growing, neurogenic tumors that arise from Schwann cells of the peripheral nerve fibers (1). They are usually located in the head/neck regions or the extremities and only rarely arise in the retroperitoneal space $(1,2)$. Retroperitoneal schwannomas (RPS) pose significant diagnostic challenges as they are frequently elusive and lack specific signs and symptoms. They have been associated with atypical presentations such as abdominal distension, hypertension, hematuria and hydronephrosis in several previously published reports (3-5). Although preoperative computed tomography (CT) and magnetic resonance imaging (MRI) may help establish the diagnosis, RPS lack specific imaging features which makes definitive diagnosis inevitably based on post-resection histopathologic evaluation and immunohistochemical labeling (5).

Herein we present a case of RPS with cystic degeneration mimicking a pancreatic cystic neoplasm. We highlight the importance of novel molecular techniques that aid in the diagnosis of this rare neoplasm. We present the following case in accordance with the CARE reporting checklist (available at https://jovs.amegroups.com/article/ view/10.21037/jovs-20-165/rc).

\section{Case presentation}

A 76-year-old male was diagnosed with an incidental pancreatic cystic lesion during workup for hematuria. He denied any symptoms and his physical examination was unremarkable. Endoscopic ultrasound (EUS) evaluation 

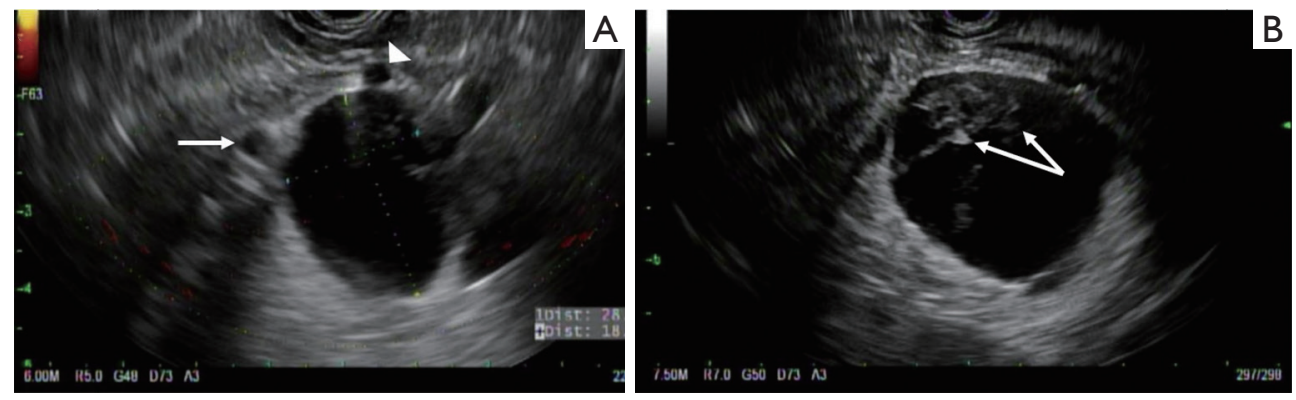

Figure 1 Endoscopic ultrasound. (A) A peripancreatic cystic lesion measuring $2.8 \mathrm{~cm} \times 1.8 \mathrm{~cm}$ with internal debris abutting the middle colic artery (white arrowhead) and a branch of the superior mesenteric vein (white arrow). (B) On 2-year follow-up, interval growth of peripancreatic cystic lesion was noted, now demonstrating increased septations and internal debris (white arrows).

revealed an anechoic lesion that seemed to arise from the uncinate process of the pancreas measuring $2.8 \mathrm{~cm}$ $\times 1.8 \mathrm{~cm}$ (Figure 1A). The cyst was composed of a single compartment without septae, lacked a solid component and was not in obvious communication with the pancreatic duct. Several other smaller cystic lesions were seen throughout the pancreas. On fine needle aspiration (FNA), golden serous fluid was aspirated. Cytology revealed scant epithelial cells with no evidence of malignancy. The amylase level in the cystic fluid was $67 \mathrm{U} / \mathrm{L}$ and the carcinoembryonic antigen (CEA) level was $493 \mathrm{ng} / \mathrm{mL}$. Molecular analysis utilizing amplification-based targeted next generation sequencing (NGS) (PancreaSeqV1) revealed no genetic alteration in key pancreatic-cancer related genes (6). On imaging, the cyst measured $2.1 \mathrm{~cm} \times 1.8 \mathrm{~cm}$. Given the aforementioned findings, a pancreatic cystic neoplasm was the most plausible diagnosis. As the patient was asymptomatic and the cyst lacked any worrisome features, annual follow-up was recommended.

On follow-up EUS 2 years after the initial diagnosis, the cyst grew significantly in size $(4.5 \mathrm{~cm} \times 4.1 \mathrm{~cm}$ in greatest dimension). It was also noted to be compartmentalized by thick septations with internal debris (Figure $1 B)$. On triphasic CT scan (Figure 2A,B) and MRI (Figure 2C,D), the growing cyst seemed to originate from the uncinate process as an exophytic outgrowth and was abutting the superior mesenteric artery and vein, as well as their proximal branches (middle colic artery and first jejunal vein, respectively). Given the likely mucinous histology posing a risk of future dedifferentiation in the setting of progressive growth, and the unfavorable location intertwined between the mesenteric vasculature, resection over continued close surveillance was advised.

On exploratory laparotomy, the cystic lesion was seen to be arising from the retroperitoneum and was only abutting the pancreas. Furthermore, no evidence of direct invasion into adjacent visceral structures or the mesenteric vasculature was identified, thus complete enucleation was accomplished. On final histopathology evaluation (Figure 3A,B) clusters of compact elongated spindle cells were seen and immunohistochemical (IHC) stains revealed positive SOX10, weakly positive S100, and negative chromogranin and CK20. On targeted NGS analysis $\left(\right.$ OncoMine $\left.{ }^{\mathrm{TM}}\right)(7)$, a genetic mutation involving both NF2 (p.G161Dfs*13) and SMARCB1 (p.R377Hc), and a copy number loss at NF2 22q12.2 were identified. These aforementioned findings support the diagnosis of a cystic peripheral nerve sheath tumor (schwannoma).

The patient had an uneventful postoperative course and was discharged home in a stable condition. He was seen in the clinic one week postoperatively and was doing well.

All procedures performed in this study were in accordance with the ethical standards of the institutional and/or national research committee(s) and with the Helsinki Declaration (as revised in 2013). Oral informed consent was obtained from the patient as he was not physically available to provide written consent due to the COVID-19 pandemic.

\section{Discussion}

Schwannomas are benign peripheral nerve sheath tumors that arise from differentiated Schwann cells most commonly in the extremities and rarely in the retroperitoneum (1-3). In our case, the schwannoma mimicked a cystic neoplasm arising from the pancreatic parenchyma. Due to the progressive growth, critical anatomic location, and the inherent risk of future dedifferentiation of- 

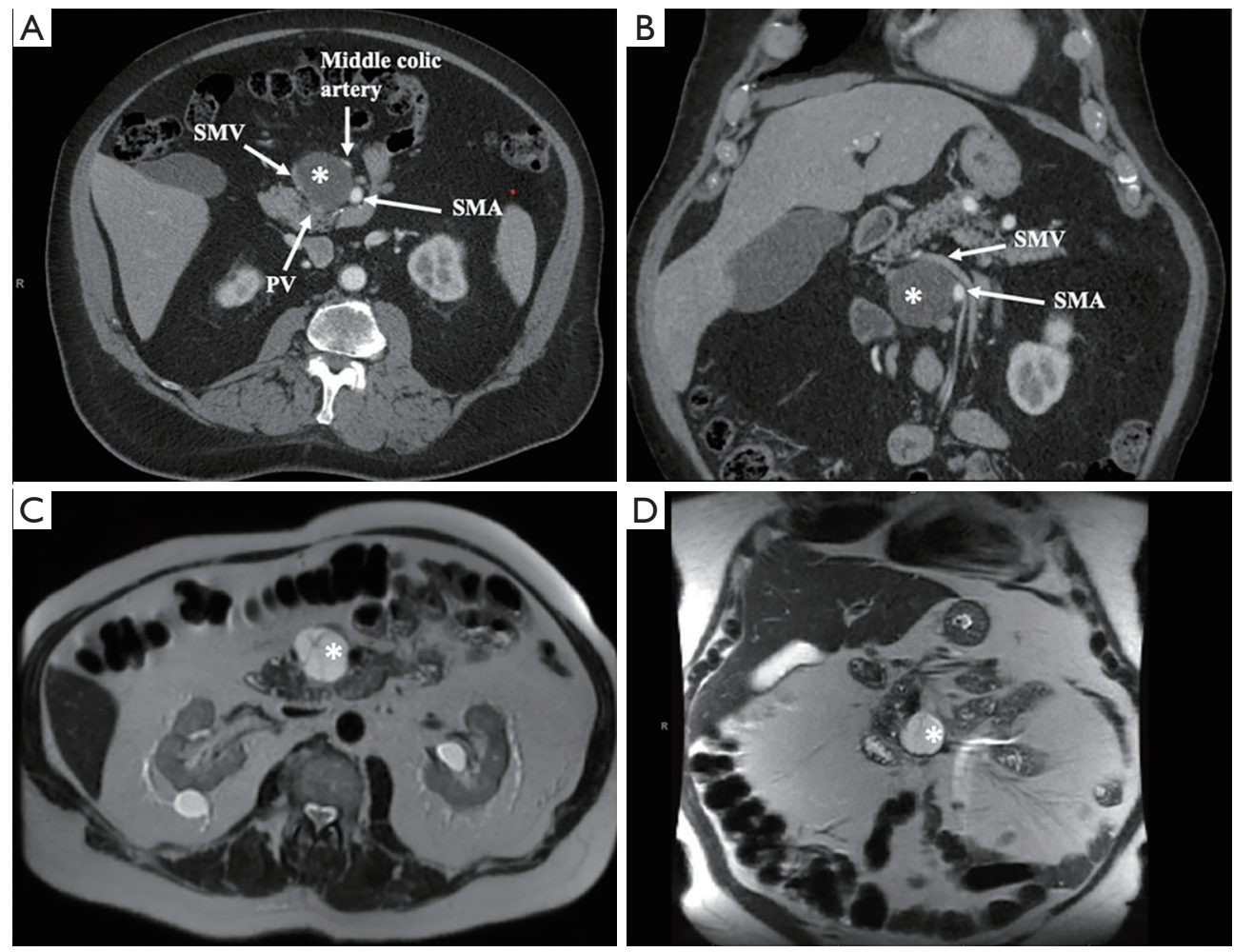

Figure 2 Computed tomography (CT) scan of the abdomen with arterial enhancement and abdominal T2-weighted MRI with fat suppression. (A) CT scan axial section depicting a peripancreatic cystic lesion (white asterisk) abutting the proximal portal vein (PV) the superior mesenteric vein (SMV), the middle colic artery, and the superior mesenteric artery (SMA). (B) CT scan coronal section demonstrating a peripancreatic cystic lesion (white asterisk) abutting and displacing the SMV and abutting the SMA. (C) Axial MRI section depicting a septated peripancreatic cystic lesion (white asterisk) abutting the caudal and medial edge of the pancreatic uncinate process. (D) Coronal MRI section demonstrating a septated peripancreatic cystic lesion (white asterisk) abutting the caudal and medial edge of the pancreatic uncinate process.

what was thought to be-a pancreatic cystic neoplasm, surgical resection was performed. A cystic degeneration of a retroperitoneal schwannoma is a rare entity, and this diagnosis can be insidious and elusive requiring a high index of suspicious.

We initially performed a EUS guided FNA and applied a pancreas targeted NGS assay (PancreaSeqV1) capable of detecting genes known to be frequently mutated in pancreatic cystic lesions (KRAS, GNAS, TP53, PIK3CA, etc.) (6) which revealed the absence of any genomic alterations. Nevertheless, the clinical characteristic and the high level of CEA in the FNA fluid suggested the presence of a mucinous lesion, while the lack of mutation on PancreaSeqV1 was rationalized as a possible false negative. In retrospect, the elevated CEA level could have been obtained from one of the adjacent intrapancreatic cystic lesions that was probably sampled along the needle track.

Postsurgical histopathology evaluation by IHC was positive for S100 and SOX10 and lacked staining for chromogranin which is highly suggestive for a schwannoma. Ultimately, genomic analysis of the excised surgical specimen was performed using Oncomine ${ }^{\mathrm{TM}}$ a comprehensive targeted NGS assay that analyses 161 genes including those that have been shown to be mutated in a subset of schwannomas tumors such as NF2 and SMARCB1-thus ascertaining the presence of a peripheral nerve sheath tumor $(7,8)$.

Although rarely, peripheral nerve sheath tumors can arise in the retroperitoneum and can have an insidious presentation. The findings presented here describe an atypical presentation of a cystic schwannoma and employ novel molecular techniques to improve diagnostic accuracy. 

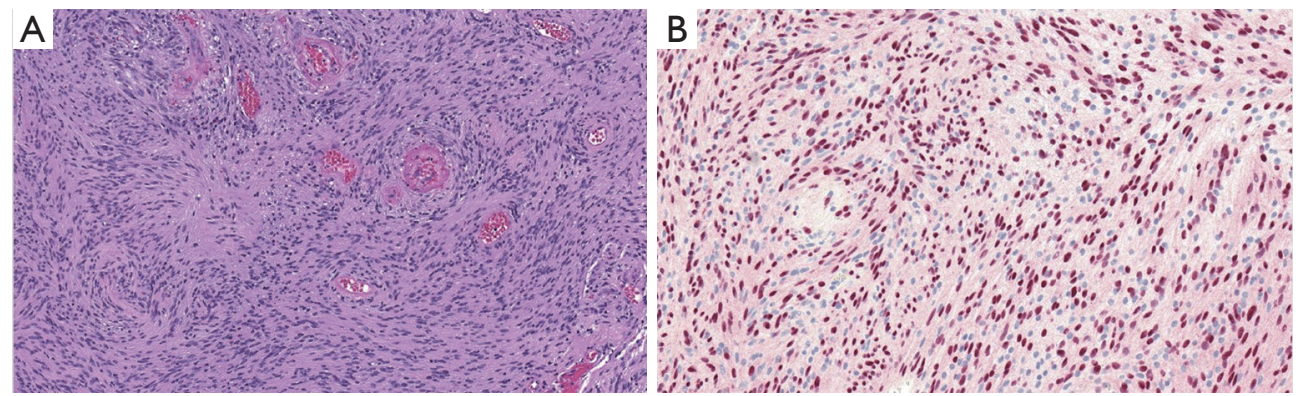

Figure 3 Histopathology of retroperitoneal schwannoma. (A) Compact elongated spindle shaped cells in a typical palisading arrangement. No atypical large nuclei or mitosis were observed (hematoxylin-eosin, $\times 200$ ). (B) Immunohistochemical staining for SOX10 shows intact expression with red nuclear staining $(\times 400)$.

\section{Acknowledgments}

Funding: None.

\section{Footnote}

Reporting Checklist: The authors have completed the CARE reporting checklist. Available at https://jovs.amegroups. com/article/view/10.21037/jovs-20-165/rc

Conflicts of Interest: All authors have completed the ICMJE uniform disclosure form (available at https://jovs. amegroups.com/article/view/10.21037/jovs-20-165/coif). The authors have no conflicts of interest to declare.

Ethical Statement: The authors are accountable for all aspects of the work in ensuring that questions related to the accuracy or integrity of any part of the work are appropriately investigated and resolved. All procedures performed in this study were in accordance with the ethical standards of the institutional and/or national research committee(s) and with the Helsinki Declaration (as revised in 2013). Oral informed consent was obtained from the patient as he was not physically available to provide written consent due to the COVID-19 pandemic.

Open Access Statement: This is an Open Access article distributed in accordance with the Creative Commons Attribution-NonCommercial-NoDerivs 4.0 International License (CC BY-NC-ND 4.0), which permits the noncommercial replication and distribution of the article with the strict proviso that no changes or edits are made and the original work is properly cited (including links to both the formal publication through the relevant DOI and the license).
See: https://creativecommons.org/licenses/by-nc-nd/4.0/.

\section{References}

1. Goh BK, Tan YM, Chung YF, et al. Retroperitoneal schwannoma. Am J Surg 2006;192:14-8.

2. Song JY, Kim SY, Park EG, et al. Schwannoma in the retroperitoneum. J Obstet Gynaecol Res 2007;33:371-5.

3. Holbrook C, Saleem N. Retroperitoneal schwannoma: an unusual cause of abdominal distention. BMJ Case Rep 2017;2017:bcr2017220221.

4. Vijayan SK, Shetty S, Bhat SR, et al. Retroperitoneal schwannoma: an atypical presentation. J Clin Diagn Res 2014;8:ND22-3.

5. Zhang L, Gao M, Zhang T, et al. Surgical management of retroperitoneal schwannoma complicated with severe hydronephrosis: A case report. Medicine (Baltimore) 2018;97:e12528.

6. Singhi AD, McGrath K, Brand RE, et al. Preoperative next-generation sequencing of pancreatic cyst fluid is highly accurate in cyst classification and detection of advanced neoplasia. Gut 2018;67:2131-41.

7. Rhodes DR, Yu J, Shanker K, et al. ONCOMINE: a cancer microarray database and integrated data-mining platform. Neoplasia 2004;6:1-6.

8. Abdellatif E. Schwannoma. PathologyOutlines.com website. Available online: http://www.pathologyoutlines.com/topic/ softtissueschwannoma.html. Accessed October 20, 2020.

doi: 10.21037/jovs-20-165

Cite this article as: AlMasri S, Nassour I, Singhi AD, Zureikat A, Paniccia A. Retroperitoneal schwannoma mimicking a pancreatic cystic neoplasm: a case report. J Vis Surg 2021;7:33. 